\title{
6-sialyllactose ameliorates dihydrotestosterone-induced benign prostatic hyperplasia through suppressing VEGF-mediated angiogenesis
}

\author{
Eun-Yeong Kim ${ }^{1,2, \#}$, Bo-Ram Jin ${ }^{3, \#}$, Tae-Wook Chung ${ }^{2}$, Sung-Jin Bae ${ }^{2}$, Hyerin Park ${ }^{1,2}$, Dongryeol Ryu ${ }^{4}$, Ling Jin ${ }^{1,2}$, \\ Hyo-Jin $\mathrm{An}^{3, *}$ \& Ki-Tae $\mathrm{Ha}^{1,2, *}$ \\ ${ }^{1}$ Department of Korean Medical Science, School of Korean Medicine and ${ }^{2}$ Korean Medical Research Center for Healthy Aging, Pusan \\ National University, Yangsan 50612, ${ }^{3}$ Department of Pharmacology, College of Korean Medicine, Sangji University, Wonju 26339, \\ ${ }^{4}$ Department of Molecular Cell Biology, Sungkyunkwan University School of Medicine, Suwon 16419, Korea
}

Benign prostatic hyperplasia (BPH), a common disease in elderly males, is accompanied by non-malignant growth of prostate tissues, subsequently causing hypoxia and angiogenesis. Although VEGF-related angiogenesis is one of the therapeutic targets of prostate cancer, there is no previous study targeting angiogenesis for treatment of BPH. Dihydrotestosterone (DHT)induced expressions of vascular endothelial growth factor (VEGF) in prostate epithelial RWPE-1 cells and human umbilical vascular endothelial cells (HUVECs). Conditioned media (CM) from DHT-treated RWPE-1 cells were transferred to HUVECs. Then, 6SL inhibited proliferation, VEGFR-2 activation, and tube formation of HUVECs transferred with $\mathrm{CM}$ from DHT-treated RWPE-1 cells. In the rat BPH model, $6 S \mathrm{~S}$ reduced prostate weight, size, and thickness of the prostate tissue. Formation of vessels in prostatic tissues were also reduced with $6 \mathrm{SL}$ treatment. We found that $6 \mathrm{SL}$ has an ameliorative effect on in vitro and in vivo the BPH model via inhibition of VEGFR-2 activation and subsequent angiogenesis. These results suggest that $6 \mathrm{SL}$ might be a candidate for development of novel BPH drugs. [BMB Reports 2019; 52(9): 560-565]

\section{INTRODUCTION}

Benign prostatic hyperplasia (BPH), non-cancerous hyperplastic proliferation of the prostate, is one of the most common

*Corresponding authors. Hyo-Jin An, Tel: +82-33-783-7503; Fax: +83-22-730-0660; E-mail: hjan@sj.ac.kr; Ki-Tae Ha, Tel: +82-51510-8464; Fax: +82-51-510-8420; E-mail: hagis@pusan.ac.kr ${ }^{\#}$ These authors contributed equally to this work.

https://doi.org/10.5483/BMBRep.2019.52.9.113

Received 20 April 2019, Revised 10 June 2019, Accepted 13 July 2019

Keywords: Benign prostatic hyperplasia, Dihydrotestosterone, VEGF, VEGFR-2, 6-Siallylactose diseases in elderly males (1). Several drugs targeting $\alpha 1$-adrenergic receptor $(2,3)$ and $5 \alpha$-reductase $(4)$ are the most common choices for clinical management of $\mathrm{BPH}$. However, these drugs have limitation on clinical usage because of side-effects, such as hypertension, nasal congestion, decreased libido, erectile dysfunction, or other drug-related problems (5, 6). Thus, many studies are struggling to find novel drugs targeting other molecules (7).

Despite that BPH is a benign disease, it develops from uncontrolled hyper-proliferation of the stromal and epithelium region (8). Thus, the fast growing region in $\mathrm{BPH}$ also undergoes hypoxic condition and subsequently induces angiogenesis, as like malignant prostatic cancer (9-11). Angiogenic factors, such as vascular endothelial growth factor (VEGF), are highly expressed in tissues of $\mathrm{BPH}$ and play a significant role in development and progression (12-16). Several studies have shown that androgen, a key hormone regulating $\mathrm{BPH}$, is a positive regulator for VEGF expression $(17,18)$. However, Wen et al. $(19,20)$ reported that DHT, a most potent androgen responsible for $\mathrm{BPH}$, failed to alter VEGF expression in prostate cancer cells. Thus, the exact mechanism for regulating VEGF in prostate tissues is controversial and unclear.

Sialyllactose (SL), an ingredient of human milk oligosaccharides (HMOs), plays a significant role in a variety of biological functions, such as immune regulation, anti-microbial, postnatal brain development, and prebiotics (21-23). Previously, we found that 6-sialyllactose (6SL) have anti-angiogenic effect via suppressing phosphorylation of VEGF receptor-2 (VEGFR-2) in human vascular endothelial cells (24). Thus, we supposed the hypothesis that $6 \mathrm{SL}$ reduces growth of $\mathrm{BPH}$ by inhibiting VEGF-mediated angiogenesis.

In this study, we showed that DHT increased expression of VEGFA in prostate epithelial cells and achieved subsequent paracrine angiogenic effect on vascular endothelial cells. The 6SL inhibited the phosphorylation of VEGFR-2, subsequent angiogenic features of vascular endothelial cells, and in vivo prostatic hyperplasia. These findings suggest that $6 \mathrm{SL}$ might be a novel candidate for treating $\mathrm{BPH}$. 


\section{RESULTS}

\section{Bioinformatic analysis}

As a first step for identifying actions of genes related with angiogenesis in human $\mathrm{BPH}$, we applied a bioinformatics approach with the NCBI GEO database (GSE32982) (25). Results from volcano plot analysis showed that angiogenic genes, especially VEGFA and ANPEP are prominent (Fig. 1A). GO enrichment analysis revealed that the angiogenesis pathway was significantly increased in tissues in human BPH group compared with normal control (Fig. 1B). The heatmap assayed by GO enrichment analysis also showed that several angiogenic genes, such as VEGFA, ANPEP, APOLD1, CYR61, EGF, ANGPTL4, FDZ5, ANG, FLT1, APOD, JUN, CECAM1, THBS1, ANGPT2, ROHB, PDGFRA, and PIK3CR were highly expressed in transcriptome of BPH tissue (Fig. 1C). Additionally, expression value of VEGFA in BPH tissue is much higher than that in normal and prostate cancer tissue (Fig. 1D).

\section{VEGFA expression in DHT-stimulated RWPE-1 cells activated VEGFR-2 in HUVECs}

To distinguish cells responsible for DHT-induced VEGFA expression, we treated DHT to prostate epithelial RWPE-1 cells and vascular endothelial HUVECs (26). As shown in Fig. 2A and $2 \mathrm{~B}, \mathrm{DHT}$ increased mRNA and protein levels of VEGFA

A)

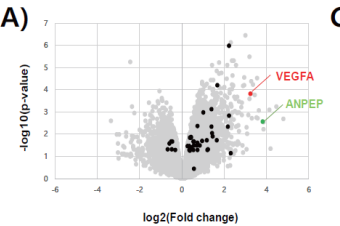

B)

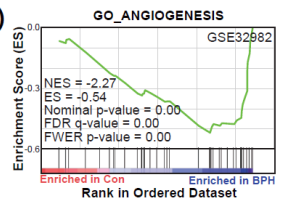

D)

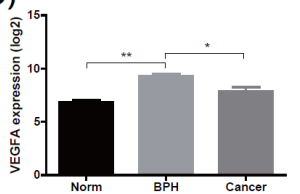

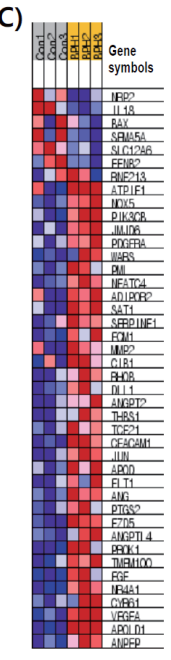

Fig. 1. Bioinformatic analysis using the transcriptomes of human prostate tissues from normal and BPH. (A) Scatter plot of DNA microarray data (GSE 32982), displaying transcript levels in normal control ( $x$ axis) and BPH (y axis) tissues. Gene sets encoding proteins related with angiogenesis are represented by red, green and black dots. (B) Heatmap analysis of genes related with angiogenesis was performed on transcriptome data from GSE32982. (C) Relative expression value (log2 transformed) of VEGFA from transcriptome of normal (Norm), BPH, and prostate cancer (Cancer) tissues are presented as means \pm SEM. $* P<$ 0.05 and $* * P<0.01$ comparing each group. only in RWPE-1 cells in a dose-dependent fashion, but not in HEVECs. Thus, to determine the paracrine effect of VEGFA secreted from prostate epithelial cells on the angiogenic features of HUVECs, we designed an in vitro experimental BPH model transferring serum from DHT-stimulated RWPE-1 toward HUVECs (Fig. S1). Viability of HUVECs with serum of DHT-stimulated RWPE-1 cells was significantly increased by dose of DHT-stimulation (Fig. 2C). Additionally, phosphorylation of VEGFR-2 and its downstream signaling, including Akt and ERK, increased by CM from DHT-stimulated RWPE-1 cells (Fig. 2D). Capillary-like tube formation of HUVECs was also evidently grown by $\mathrm{CM}$ of DHT-incubated RWPE-1 (Fig. 2E). At these concentrations, DHT does not affect viability of RWPE-1 cells and HUVECs (Fig. S2A and S2B). Thus, we set concentration of DHT at $100 \mathrm{nM}$ for consequent experiments.

6SL inhibited paracrine activation of angiogenesis in HUVECs stimulated by DHT-treated RWPE-1

Previously, we demonstrated that sialyllactose, especially 6SL,
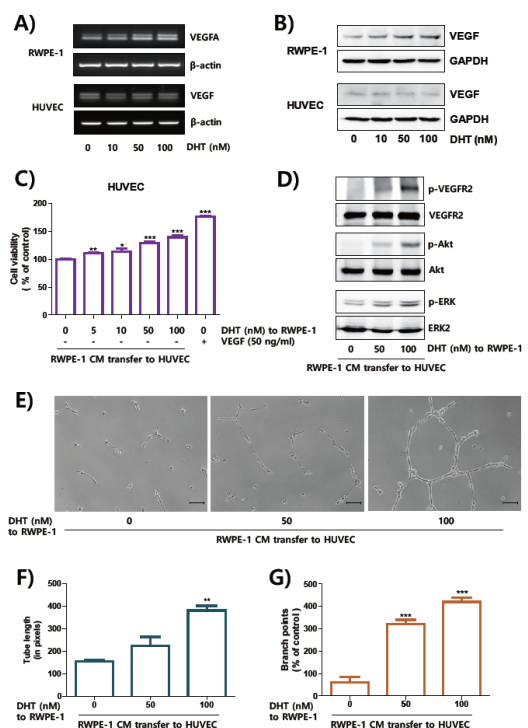

Fig. 2. The culture media from DHT-treated RWPE-1 cells induced activation of VEGFR-2 in HUVEC cells. (A, B) The RWPE-1 and HUVEC cells were incubated with DHT for 24 hours. The expressions of VEGFA were determined by RT-PCR (A) and Western blot analysis (B). The expression levels of $\beta$-actin and GAPDH were used for internal control for RT-PCR and Western blot analysis, respectively. (C) HUVECs were incubated with CM for 48 hours. For positive control, HUVECs were incubated with VEGF $(50 \mathrm{ng} / \mathrm{ml})$. Cell proliferation was measured by MTT assay and presented as means \pm SEM. (D) HUVEC cells were incubated with $\mathrm{CM}$ for one hour, signal molecules was determined by Western blot analysis. (E-G) Tube formation assay was performed at 12 hours after treatment of culture media on cells on the matrigel-coated plates, scale bar $=10 \mu \mathrm{m}$. The length of tube $(\mathrm{F})$ and numbers of branch points $(G)$ were shown as means \pm SEM. $* * \mathrm{P}<0.01$ and ${ }^{* * * P}<0.001$ compared to control. 
has anti-angiogenic action though inhibiting interaction between VEGFA and VEGFR-2 in the cancer model (24). To elucidate if $6 \mathrm{SL}$ also suppress angiogenesis in the in vitro $\mathrm{BPH}$ model, we treated 6SL on HUVECs before incubation with CM from DHT-treated RWPE-1 cells (Fig. 3A). 6SL inhibited proliferation of HUVECs induced by CM from DHT-treated RWPE-1 cells, in a dose-dependent manner (Fig. 3B). Activated phosphorylation of VEGFR-2 and its downstream signaling pathway by transferring DHT-stimulated RWPE-1 media were also diminished by $6 \mathrm{SL}$ treatment (Fig. 3C). We also examined if the capillary like-tube formation of HUVECs were inhibited by $6 \mathrm{SL}$ treatment mimicking angiogenesis in the $\mathrm{BPH}$ microenvironment. As shown in Fig. 3D-F, tube formatting morphology, tube length, and branch points of HUVECs were significantly decreased with 6SL treatment before incubating with CM from DHT-stimulated RWPE-1 cells. From these results, we assume that 6SL inhibited angiogenic features of HUVECs through inhibiting VEGFR-2 activation in the in vitro BPH model.

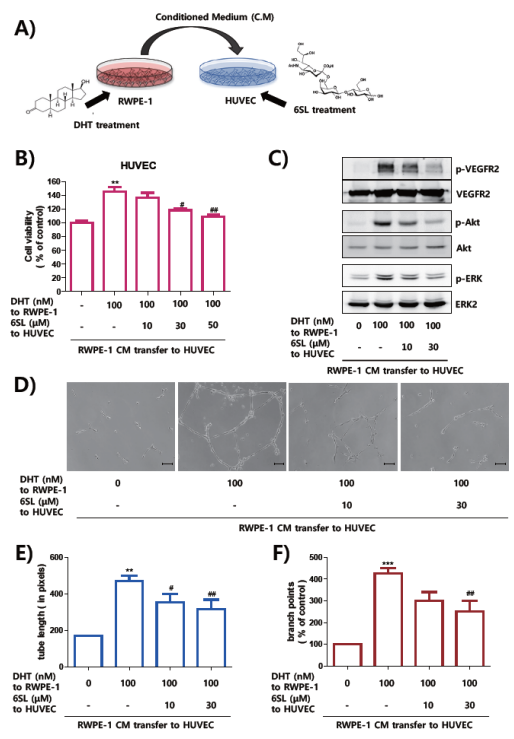

Fig. 3. 6SL inhibited angiogenesis of HUVECs induced by culture media of RWPE-1. (A) HUVEC cells were treated with 6SL and angiogenesis induced with $\mathrm{CM}$ transfer incubated with DHT of RWPE-1. (B) HUVECs were treated with indicated concentrations of 6SL for 48 hours in the presence or absence of CM for 24 hours. Effect of growth inhibition of HUVECs was measured by MTT assay and represented as means + SEM. ${ }^{*} \mathrm{P}<0.01$ compared to control (1st lane), $\mathrm{P} P<0.1$ and ${ }^{\# \#} \mathrm{P}<0.01$ compared to control group (2nd lane). (C) Cells were starved for six hours and pretreated with 6SL for one hour before stimulated with transfer of CM for one hour. Activation of signal molecules was determined by Western blot analysis. (D-F) HUVECs were incubated with sialyllactose in matrigel-coated 24-well plate and CM stimulated by $\mathrm{DHT}$ for 24 hours, scale bar $=10 \mu \mathrm{m}$. Formation of tubes were observed with a microscope. The length of tube $(\mathrm{E})$ and numbers of branch points ( $F$ ) of HUVECs were shown as means \pm SEM. ${ }^{* * P}<0.01$ and ${ }^{* * * P}<0.001$ compared to control (1st lane) and ${ }^{\#} \mathrm{P}<0.01$ compared to positive control (2nd lane).

\section{SL inhibited angiogenesis and hyperplasia in prostatic tissues of rat BPH model}

To test if $6 \mathrm{SL}$ could reduce growth of prostate tissues, we adopted the chronic testosterone-treated rat model, which has been used to assess drugs targeting BPH (27). In Fig. 4A, tissues from the BPH group showed swollen prostate and increased supply of blood vessels. However, the major macroscopic parameter of testosterone-treated prostate tissues was ameliorated by $10 \mathrm{mg} / \mathrm{kg}$ of $6 \mathrm{SL}, 40 \mathrm{mg} / \mathrm{kg}$ of $6 \mathrm{SL}$ and Saw Palmetto (SP), as positive control, commonly recommended as alternative therapeutic of BPH (28-30). Factors, including prostate weight, relative prostate weight, and PW/BW ratio, which was increased in the $\mathrm{BPH}$ group, were all down-regulated in the SP, $10 \mathrm{mg} / \mathrm{kg}$ of $6 \mathrm{SL}$, and $40 \mathrm{mg} / \mathrm{kg}$ of 6SL-treated groups (Fig. 4A-D). Additionally, as shown in Fig $4 \mathrm{E}$ and $4 \mathrm{~F}$, the $\mathrm{H} \& \mathrm{E}$ staining and histological evaluation (TEPT) of prostate tissues clearly demonstrated that the rats with $\mathrm{BPH}$ showed increased thickness of the epithelium, constricted glandular lumen area, and typical pattern of hyperplasia. However, these morphological changes in prostate tissues induced by chronic testosterone treatment were ameliorated by treatment of SP, $10 \mathrm{mg} / \mathrm{kg}$ of $6 \mathrm{SL}$, and $40 \mathrm{mg} / \mathrm{kg}$ of $6 \mathrm{SL}$. Also, the anti-angiogenic effects of $6 \mathrm{SL}$ was investigated by

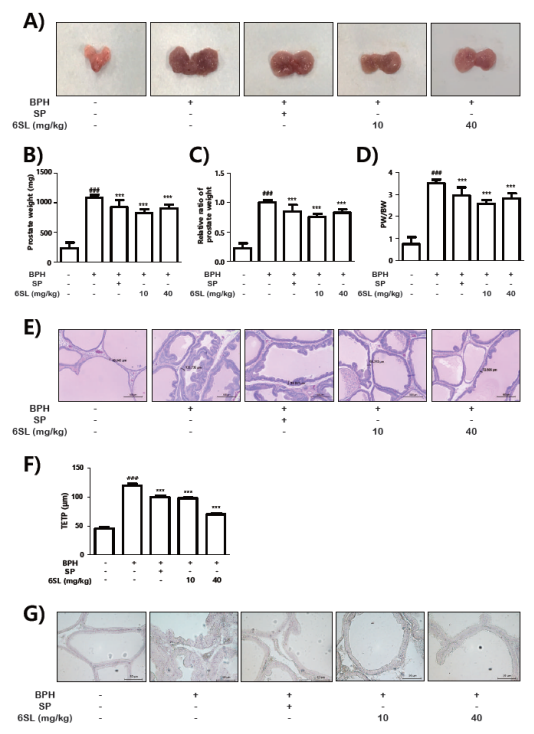

Fig. 4. $6 \mathrm{SL}$ reduced prostate growth and histological degree in rats with the $\mathrm{BPH}$ model. Prostate hyperplasia induced by $\mathrm{BPH}$ were treated 6SL or finasteride by oral administration for four weeks. Representative photographs (A). Total prostate weight of the rat (B), relative ratio of prostate weight $(\mathrm{C})$ and $\mathrm{PW} / \mathrm{BW}$ ratio (D) was assessed for the control, rat with $\mathrm{BPH}, \mathrm{SP}$, and 6SL groups. (E) $\mathrm{H} \& \mathrm{E}$ staining of prostate tissue from rats with $\mathrm{BPH}$ models; original magnification $40 \times$. (F) TEPT was measured at the end of the experiment. Results were presented as mean \pm SEM. \#\# $\mathrm{P}<0.001$ compared to control (1st lane), $* * * \mathrm{P}<0.001$ compared to positive control (2nd lane). (G) Prostate tissues were immunostained with CD31 antibody to detect newly formed vessels. 
IHC analysis of CD31 expression, a marker of neovascularization. Increased CD31 expression was observed in the BPH group, while $10 \mathrm{mg} / \mathrm{kg}$ of $6 \mathrm{SL}$ and $40 \mathrm{mg} / \mathrm{kg}$ of $6 \mathrm{SL}$-treated groups showed significant decrease of CD31 expression in comparison with that in the BPH group (Fig. 4G). SP-treated group also presented reduction of CD31-stained vascularization. Collectively, these results presented that $6 \mathrm{SL}$ had anti-angiogenic and anti-hyperplastic effect in the in vivo BPH model.

\section{DISCUSSION}

Although their significant benefit of $5 \alpha$-reductase inhibitors and natural products (28-30), there are several reports raising clinical concerns because of their small effective size and risk of adverse effect including sexual complications (31-33). Here, we confirmed that genes related angiogenesis pathways, such as ANPEP, APOLD1, and VEGFA, were elevated in tissues of $\mathrm{BPH}$ patients (Fig. 1). Among these genes, VEGFA was most highly expressed in BPH tissues compared with that in prostatic cancer as well as in normal. As these results show a good correlation with previous studies $(34,35)$, we hypothesized that inhibition of angiogenesis may be an alternative therapeutic option.

Previously, expression of VEGF was confirmed in epithelial cells and stromal cells of BPH specimens $(15,36)$. The level of VEGF immunostaining is higher in BPH epithelial cells compared with that in stromal cells, even in prostate cancer tissues $(24,36,37)$. Although there is some controversy (20), the androgen including DHT is regarded as an inducing factor for VEGF expression $(17,18,38)$. In this study, we detected expression of VEGFA in DHT-stimulated prostatic epithelial cells but not in vascular endothelial cells (Fig. 2A). The paracrine effect of VEGF secreted in DHT-stimulated CM of prostatic epithelial cells was also confirmed (Fig. 2B-G).

Here, we applied 6SL, previously reported inhibitor of VEGFR-2 activation (24), to block the VEGF-related angiogenesis in in vitro and in vivo BPH models. 6SL is an acidic component of $\mathrm{HMOs}$ harboring diverse biological actions, including cell-pathogen interaction, immune response, brain development, and tumor angiogenesis. In this study, 6SL suppressed proliferation and tube-like morphological changes of vascular endothelial cells via inhibiting activation of VEGFR-2 and its downstream signaling pathway (Fig. 3). Additionally, in the rat model, 6SL successfully reduced growth of prostate by means of gross and histological observations (Fig. 4). Neovascularization, stained by CD31, was also reduced by $6 \mathrm{SL}$ treatment. The effect of $6 \mathrm{SL}$ is superior by means of its lower concentration, almost lesser than 2.5 fold, and anti-angiogenic property.

In conclusion, we found that $6 \mathrm{SL}$ has inhibitory effect on DHT-stimulated paracrine angiogenesis through suppressing VEGFR-2 activation. 6SL suppressed proliferation, VEGFR-2 signaling pathways, and tube formation of vascular endothelial cells increased by transferring CM from DHT-treated prostatic epithelial cells. In vivo experiments, 6SL effectively reduced growth of prostatic tissues and formation of new vessels. From these results, we suggest that $6 \mathrm{SL}$ might be a potential candidate for development of a novel drug for treating $\mathrm{BPH}$ via inhibition of angiogenesis.

\section{MATERIALS AND METHODS}

\section{Materials}

6SL were purchased from Carbosynth Ltd. (Berkshire, UK). Antibodies against phosphorylated and total form of VEGFR-2, Akt and ERK were purchased from Cell Signaling Technology (Danvers, MA). Testosterone propionate (TP) was purchased from Wako Pure Chemicals (Tokyo, Japan). Saw palmetto extract (SP) was obtained from Chongkundang Pharm. (Seoul, Korea). Antibodies against cluster of differentiation 31 (CD31; sc-376764) was purchased from Santa Cruz Biotechnology (Santa Cruz, CA).

\section{Bioinformatic analysis}

Volcano plot analysis was performed as previously described (39) using transcriptomes of human prostate tissues from normal castrated, benign hyperplasia, and cancer patients (NCBI GEO dataset, GSE32982) (25). Gene Ontology (GO) enrichment analysis (http://geneontology.org/) was built also using transcriptomes of human prostate tissue (GSE32982) as described in the previous study (40). Expression values of VEGFA were collected from GEO2R analysis of GSE32982 (Sample ID=210513_s_at).

\section{Cell culture}

RWPE-1 cells were purchased form American Type Culture Collection (Manassas, VA) and cultured in keratinocyte serum free medium (K-SFM) from ThermoFischer Scientific (Waltham, MA). Human umbilical vein endothelial cells (HUVECs) were purchased from Cambrex Inc. (Walkersville, MD) and cultured in endothelial growth medium-2 (EGM-2; Cambrex Inc.) All cell lines were incubated at $37^{\circ} \mathrm{C}$ in a humidified $5 \% \mathrm{CO}_{2}$ cell culture incubator.

\section{Cell viability}

Cells were incubated in 24-well plates with indicated concentrations of $6 \mathrm{SL}$ or DHT for 24 hours. To examined growth inhibition by 6SL, HUVECs were cultured indicated dose of $6 \mathrm{SL}$ and/or conditioned medium (CM) from DHT-treated prostate epithelial cells for 48 hours. Then, MTT solution $(0.5 \mathrm{mg} / \mathrm{ml})$ was incubated for four hours at $37^{\circ} \mathrm{C}$ in a $\mathrm{CO}_{2}$ incubator, the formazan crystal were dissolved in $300 \mu \mathrm{l}$ of ethanol:DMSO $(\mathrm{v} / \mathrm{v}, 1: 1)$ and estimated by measuring absorbance at $540 \mathrm{~nm}$ with a microplate reader (Spectramax M2; Molecular Devices, San Jose, CA).

\section{Western blot analysis}

Total proteins from HUVECs using 1\% NP-40 lysis buffer. 
Equal amounts of proteins were used for Western blot analysis and membrane were incubated with indicated primary antibodies. To detect target proteins, the membrane was examined with a Pierce ECL plus (ThermoFischer Scientific) using ImageQuant LAS4000 (GE Healthcare, Pittsburgh, PA).

\section{Tube forming assay}

A capillary-like tube formation of HUVECs was perfomed with Matrigel-coated 24-well (41). Matrigel (BD Bioscience, San Jose, CA) was mixed with EGM-2 medium and added to each well of the 24-well culture plates. For preparation of conditioned media, RWPE-1 cells $\left(5 \times 10^{5}\right)$ suspended in 1.5 $\mathrm{ml}$ of K-SFM were seeded in six-well plates, treated with DHT for 24 hours. After incubation, $1 \mathrm{ml}$ of conditioned medium was harvested and mixed with HUVECs, $1 \%$ fetal bovine serum (FBS; Sigma-Aldrich) and with or without 6SL. And the mixture was added to 24-well culture plates coated with matrigel at $37^{\circ} \mathrm{C}$. After 24 hours of incubation, tube formation of each well was photographed with a light microscope (ECLIPSE TS100; Nikon, Tokyo, Japan).

\section{Animals}

All animal experimental procedures were performed on six weeks old male Sprague-Dawley rats (Daehan Biolink Co., Daejeon, Korea) under the Institutional Animal Care and Use Committee (IACUC) of Sangji University before the initiation of animal study (\#2018-25). First, anesthetization with zoletil 50 , testicles were removed from rats of the $\mathrm{BPH}$ model and after the recovery period, experimental rats of the $\mathrm{BPH}$ group were injected with testosterone propionate $(10 \mathrm{mg} / \mathrm{kg})$. Rats of the BPH model were orally administrated with or without $6 \mathrm{SL}$ or SP $(100 \mathrm{mg} / \mathrm{kg})$ using oral zonde for four weeks. At the end of the experiment, prostate tissues were excised, rinsed and weighed immediately after removal. PW/BW ratio was calculated using the following equation: $\mathrm{PW} / \mathrm{BW}$ ratio = (prostate weight of each animal from experimental group/body weight of each animal from the experimental group) $\times 1,000$.

\section{Hematoxylin and Eosin (H\&E) staining and histological analysis}

Tissues for H\&E staining were fixed with $10 \%$ formalin and embedded in paraffin and serially paraffin sections were cut into 4-7 $\mu \mathrm{m}$ thickness. Slides were de-paraffinized and endogenous peroxidase exhaustion was performed. Sections were stained with $\mathrm{H} \& \mathrm{E}$ for histological examination. Images were captured using a SZX10 microscope (Olympus Co., Tokyo, Japan). Thickness of epithelium tissue from prostate (TETP) was measured using Leica Application Suite (ver.3.3.0) software (Leica Biosystems, Wetzlar, Germany) for histological analysis.

\section{Immunohistochemistry (IHC)}

Tissue sections were blocked for one hour at room temperature with $10 \%$ normal goat serum (ThermoFischer Scientific) and were co-incubated with CD31 primary antibody (1:100) overnight at $4^{\circ} \mathrm{C}$. Slides were incubated with Peroxidase AffiniPure Goat Anti-mouse IgG (1:100) for one hour at room temperature, followed by incubation with H\&E used as a counterstain. Images of the IHC slides were visualized by optical microscopy (ECLIPSE Ni-U, Nikon) and digital camera (DS-Fi2, Nikon) rendered using NIS-Elements F Ver 4.00.

\section{Statistical analysis}

Values from cell viability and tube formation assay were calculated by the percentage of control cells and expressed as mean \pm Standard Error (SEM). Differences of cell viability between mean values were decided by Student's t-test and statistical analysis of tube formation assay and in vivo study were decided by one-way analysis of variance with a post hoc Dunnet's comparison using GraphPad Prism (GraphPad Software, San Jose, CA). All the experiments were performed at least three times, independently.

\section{ACKNOWLEDGEMENTS}

This study was supported by a grant from the National Research Foundation of Korea (NRF) funded by the Ministry of Science and ICT, of the Korean Government (Grant no. NRF-2014R1A5A20009936 to K.T.H.) and Basic Science Research Program through the National Research Foundation of Korea (NRF) funded by the Ministry of Science, ICT \& Future Planning (NRF-2016R1C1B2011827 to H.J.A.).

\section{CONFLICTS OF INTEREST}

The authors have no conflicting interests.

\section{REFERENCES}

1. Emberton M, Andriole GL, de la Rosette J et al (2003) Benign prostatic hyperplasia: a progressive disease of aging men. Urology 61, 267-273

2. O'Leary MP (2003) Lower urinary tract symptoms/benign prostatic hyperplasia: maintaining symptom control and reducing complications. Urology 62, 15-23

3. Keledjian K, Borkowski A, Kim G et al (2001) Reduction of human prostate tumor vascularity by the alpha1adrenoceptor antagonist terazosin. Prostate 48, 71-78

4. Carson C 3rd, Rittmaster R (2003) The role of dihydrotestosterone in benign prostatic hyperplasia. Urology 61, 2-7

5. Zaman Huri H, Hui Xin C, Sulaiman CZ (2014) Drug-related problems in patients with benign prostatic hyperplasia: a cross sectional retrospective study. PLoS One 9, e86215

6. DuBeau CE, Resnick NM (1992) Controversies in the diagnosis and management of benign prostatic hypertrophy. Adv Intern Med 37, 55-83

7. Chung KJ, Chung BI (2017) Expected next-generation drugs under development in relation to voiding 
symptoms. Int Neurourol J 21, 97-101

8. McNeal JE (1978) Origin and evolution of benign prostatic enlargement. Invest Urol 15, 340-345

9. Lekas A, Lazaris AC, Deliveliotis C et al (2006) The expression of hypoxia-inducible factor-1alpha (HIF-1alpha) and angiogenesis markers in hyperplastic and malignant prostate tissue. Anticancer Res 26, 2989-2993

10. Wu F, Ding S, Li X et al (2016) Elevated expression of HIF-lalpha in actively growing prostate tissues is associated with clinical features of benign prostatic hyperplasia. Oncotarget 7, 12053-12062

11. Pareek G, Shevchuk M, Armenakas NA et al (2003) The effect of finasteride on the expression of vascular endothelial growth factor and microvessel density: a possible mechanism for decreased prostatic bleeding in treated patients. J Urol 169, 20-23

12. Stefanou D, Batistatou A, Kamina S, Arkoumani E, Papachristou DJ, Agnantis NJ (2004) Expression of vascular endothelial growth factor (VEGF) and association with microvessel density in benign prostatic hyperplasia and prostate cancer. In Vivo 18, 155-160

13. Aweimer A, Stachon T, Tannapfel A, Köller M, Truss MC, Stachon A (2012) Regulation of soluble VEGFR-2 secreted by microvascular endothelial cells derived from human BPH. Prostate Cancer Prostatic Dis 15, 157-164

14. Berger AP, Kofler K, Bektic J et al (2003) Increased growth factor production in a human prostatic stromal cell culture model caused by hypoxia. Prostate 57, 57-65

15. Jackson MW, Bentel JM, Tilley WD (1997) Vascular endothelial growth factor (VEGF) expression in prostate cancer and benign prostatic hyperplasia. J Urol 157, 2323-2328

16. Lekas AG, Lazaris AC, Chrisofos M et al (2006) Finasteride effects on hypoxia and angiogenetic markers in benign prostatic hyperplasia. Urology 68, 436-441

17. Sordello S, Bertrand N, Plouët J (1998) Vascular endothelial growth factor is up-regulated in vitro and in vivo by androgens. Biochem Biophys Res Commun 251, 287-290

18. Levine AC, Liu XH, Greenberg PD et al (1998) Androgens induce the expression of vascular endothelial growth factor in human fetal prostatic fibroblasts. Endocrinology $139,4672-4678$

19. Foo KT (2017) Pathophysiology of clinical benign prostatic hyperplasia. Asian J Urol 4, 152-157

20. Wen J, Zhao Y, Li J et al (2013) Suppression of DHT-induced paracrine stimulation of endothelial cell growth by estrogens via prostate cancer cells. Prostate 73 , 1069-1081

21. Wu S, Grimm R, German JB, Lebrilla CB (2011) Annotation and structural analysis of sialylated human milk oligosaccharides. J Proteome Res 10, 856-868

22. Yonekawa T, Malicdan MC, Cho A et al (2014) Sialyllactose ameliorates myopathic phenotypes in symptomatic GNE myopathy model mice. Brain 137, 2670-2679

23. Bode L (2012) Human milk oligosaccharides: every baby needs a sugar mama. Glycobiology 22, 1147-1162

24. Chung TW, Kim EY, Kim SJ et al (2017) Sialyllactose suppresses angiogenesis by inhibiting VEGFR-2 activation, and tumor progression. Oncotarget 8, 58152-58162

25. Vaarala MH, Hirvikoski P, Kauppila S, Paavonen TK
(2012) Identification of androgen-regulated genes in human prostate. Mol Med Rep 6, 466-472

26. Park SA, Jeong MS, Ha KT, Jang SB (2018) Structure and function of vascular endothelial growth factor and its receptor system. BMB Rep 51, 73-78

27. Li J, Tian Y, Guo S, Gu H, Yuan Q, Xie X (2018) Testosterone-induced benign prostatic hyperplasia rat and dog as facile models to assess drugs targeting lower urinary tract symptoms. PLoS One 13, e0191469

28. Kim SK, Seok H, Park HJ et al (2015) Inhibitory effect of curcumin on testosterone induced benign prostatic hyperplasia rat model. BMC Complement Altern Med 15, 380

29. Minutoli L, Bitto A, Squadrito F et al (2013) Serenoa repens, lycopene and selenium: a triple therapeutic approach to manage benign prostatic hyperplasia. Curr Med Chem 20, 1306-1312

30. Said MM, Hassan NS, Schlicht MJ, Bosland MC (2015) Flaxseed suppressed prostatic epithelial proliferation in a rat model of benign prostatic hyperplasia. J Toxicol Environ Health A 78, 453-465

31. Kim JH, Baek MJ, Sun HY et al (2018) Efficacy and safety of 5 alpha-reductase inhibitor monotherapy in patients with benign prostatic hyperplasia: A meta-analysis. PLoS One 13, e0203479

32. Jun JEJ, Kinkade A, Tung ACH, Tejani AM (2017) 5alpha-Reductase inhibitors for treatment of benign prostatic hyperplasia: A systematic review and metaanalysis. Can J Hosp Pharm 70, 113-119

33. Thomas D, Chughtai B, Kini M, Te A (2017) Emerging drugs for the treatment of benign prostatic hyperplasia. Expert Opin Emerg Drugs 22, 201-212

34. Bostwick DG, Iczkowski KA (1998) Microvessel density in prostate cancer: prognostic and therapeutic utility. Semin Urol Oncol 16, 118-123

35. Padhani AR, Harvey CJ, Cosgrove DO (2005) Angiogenesis imaging in the management of prostate cancer. Nat Clin Pract Urol 2, 596-607

36. Ravindranath N, Wion D, Brachet P, Djakiew D (2001) Epidermal growth factor modulates the expression of vascular endothelial growth factor in the human prostate. J Androl 22, 432-443

37. ten Bruggencate SJ, Bovee-Oudenhoven IM, Feitsma AL, van Hoffen E, Schoterman MH (2014) Functional role and mechanisms of sialyllactose and other sialylated milk oligosaccharides. Nutr Rev 72, 377-389

38. Godoy A, Montecinos VP, Gray DR et al (2011) Androgen deprivation induces rapid involution and recovery of human prostate vasculature. Am J Physiol Endocrinol Metab 300, E263-275

39. Chung HK, Ryu D, Kim KS et al (2017) Growth differentiation factor 15 is a myomitokine governing systemic energy homeostasis. J Cell Biol 216, 149-165

40. Ryu D, Jo YS, Lo Sasso G et al (2014) A SIRT7-dependent acetylation switch of GABPbeta1 controls mitochondrial function. Cell Metab 20, 856-869

41. Chung TW, Kim SJ, Choi HJ et al (2013) CAPE suppresses VEGFR-2 activation, and tumor neovascularization and growth. J Mol Med (Berl) 91, 271-282 